\title{
Application of Innovative Geochemical and Mineralogical Techniques to Understanding the La Victoria Epithermal Gold Deposit, Peru
}

\author{
Kelsey L. Rozon ${ }^{1}$, Neil R. Banerjee ${ }^{1 *}$, Lisa L. Van Loon ${ }^{1,2}$ and Bill Pearson ${ }^{4}$ \\ 1. Department of Earth Sciences, Western University, London, ON, Canada. \\ 2. LISA CAN Analytical Solutions Inc., Saskatoon, SK, Canada. \\ 3. Eloro Resources Ltd., Toronto, ON, Canada. \\ * Corresponding author: neil.banerjee@uwo.ca
}

The La Victoria property is located along an epithermal gold belt in the Ancash Region of the western Peruvian Andes that is host to many world class gold deposits. The mineralogy at La Victoria is tantalizingly similar to other major ore deposits in the area: specifically, the presence of breccias, vuggy quartz, sericite, covellite, arsenopyrite and sphalerite. Two major mineralized areas (San Markito and Rufina) have been identified based on initial exploration techniques such as zone mapping and sampling, mineral mapping, geophysical surveys, and petrography. Thus far, both prospects seem to be related to low-sulphidation epithermal deposits, with possible porphyry copper overprinting in the Rufina area. The focus is to examine the geochemistry of grab samples from mineralized areas to determine the mineralogical and geochemical signatures of the gold mineralization and put these into a consistent depositional model, which will ultimately help target more promising areas to drill. The analytical techniques used in this study include petrographic analysis, scanning electron microscopy (SEM), electron probe micro analysis (EPMA), X-ray diffraction (XRD), Synchrotron micro X-ray fluorescence (SR- $\mu \mathrm{XRF}$ ), and micro X-ray absorption near-edge structure ( $\mu \mathrm{XANES}$ ) spectroscopy. This novel combination of techniques was used to characterize geochemistry, textures, mineralogy, and oxidation states of representative samples to better characterize the gold mineralization.

The La Victoria thin sections were prepared from surface grab samples representative of each prospect. A Nikon Eclipse LV100POL polarizing microscope at Western University was used to identify key minerals and associated assemblages. The Nikon NIS-Element D 3.2 64-bit imaging software was used to capture photomicrographs in plane polarized, transmitted and reflected light. EMPA analysis was conducted using a JEOL JXA-8530F Hyperprobe in the Earth and Planetary Materials Analysis Laboratory. Minerals of interest were first characterized using secondary electron imaging (SEI) and backscattered electron (BSE) imaging then probed using energy dispersive X-ray spectroscopy (EDS) and wavelength dispersive X-ray spectroscopy (WDS) analyses at $15 \mathrm{keV}$. Powdered samples were analyzed both using a Rigaku XRD machine at Western and at the CMCF beamline at the Canadian Light Source (CLS) in Saskatoon, SK. XRD was used to identify alteration mineralogy and major deposit characterizing minerals. Thin sections and rock offcuts were analyzed by SR- $\mu$ XRF and $\mu$ XANES spectroscopy at the VESPERS beamline at the CLS, and the F3 beamline at the Cornell High Energy Synchrotron Source (CHESS) in Ithaca, NY, to create 2D trace element maps that reveal spatial and structural associations with gold mineralization. At VESPERS, polychromatic $(5-20 \mathrm{keV})$ pink beam with a spot size of $3 \mu \mathrm{m} \times 3 \mu \mathrm{m}$ was used for mapping. A Vortex®-ME4 four element silicon drift detector (SDD) was used to measure the SR- $\mu$ XRF signal while rastering across the sample in both directions with a step size of $5 \mu \mathrm{m} \times 5 \mu \mathrm{m}$ and a dwell time of 1 second. SR- $\mu$ XRF data analysis was done with Peakaboo 5.1.0 [1]. Spot Au $\mu$ XANES spectra were collected using the Si (111) monochromator with an energy resolution of $1 \mathrm{eV}$ at $10 \mathrm{keV}$. The data were collected in fluorescence mode with the detector windowed to correspond to the Au L $\alpha$ fluorescence emission peak to maximize 
the signal to noise. $\mu$ XANES data was collected over an energy range of $-200 \mathrm{eV}$ to $+380 \mathrm{eV}$ (relative to the edge), with a dwell time of $5 \mathrm{sec}$ per point and a step size of $0.5 \mathrm{eV}$ near the edge. Two scans were collected per spot. XANES data were processed using Athena [2]. At CHESS, the incident X-ray energy was set to $16.2 \mathrm{keV}$. A 4-element SDD Vortex detector with a Quantum express processor was used to measure the XRF signal with a beam spot and step size of $100 \mu \mathrm{m}$ x $100 \mu \mathrm{m}$ using a dwell time of 100 msec. High resolution mapping of select regions was then performed under similar conditions with a beam spot and step size of $20 \mu \mathrm{m}$ x $20 \mu \mathrm{m}$. Data analysis was done using pyMCA 5.3.1 [3]

The combination of novel techniques employed in this study (petrography, EMPA, XRD, SXRF and SXANES) have revealed that the La Victoria property is host to a low-sulphidation epithermal deposit that may be overprinted by intermediate- to high-sulphidation, porphyry $\mathrm{Cu}-\mathrm{Au}$, carbonate-base metal, and polymetallic silver mineralization. Gold mineralization is associated with quartz sulphide veins, sericitization and phyllic alteration. Microscopic electrum and free gold are found associated with scorodite and/or within arsenopyrite. However, synchrotron analysis has shown that the gold is metallic and not refractory, which has important implications for geometallurgy. The mineralogy at the La Victoria property is similar to other major ore deposits in the area such as La Arena, Lagunas Norte, and Shahuindo and thus has great potential to become a major ore deposit itself [4].

\section{References:}

[1] N Sherry et al., Science Studio Project Summary (2012), http://sciencestudio.net/summary.php.

[2] B Ravel, M Newville and J Synchr. Rad. 12 (2005), p. 537.

[3] VA Solé et al., Spectrochim. Acta Part B 62 (2007), p. 63.

[4] Research described in this paper was performed at the CLS, which is supported by the CFI, NSERC, the U of S, the Gov. of SK, WED Canada, the NRC, and the CIHR. Research described in this paper was performed at CHESS, which is supported by the NSF under award DMR-1332208. We thank R. Feng, P. Blanchard, and L. Smieska for their support in conducting the experiments. The authors acknowledge funding from NSERC, Mitacs, and Eloro Resources Ltd.

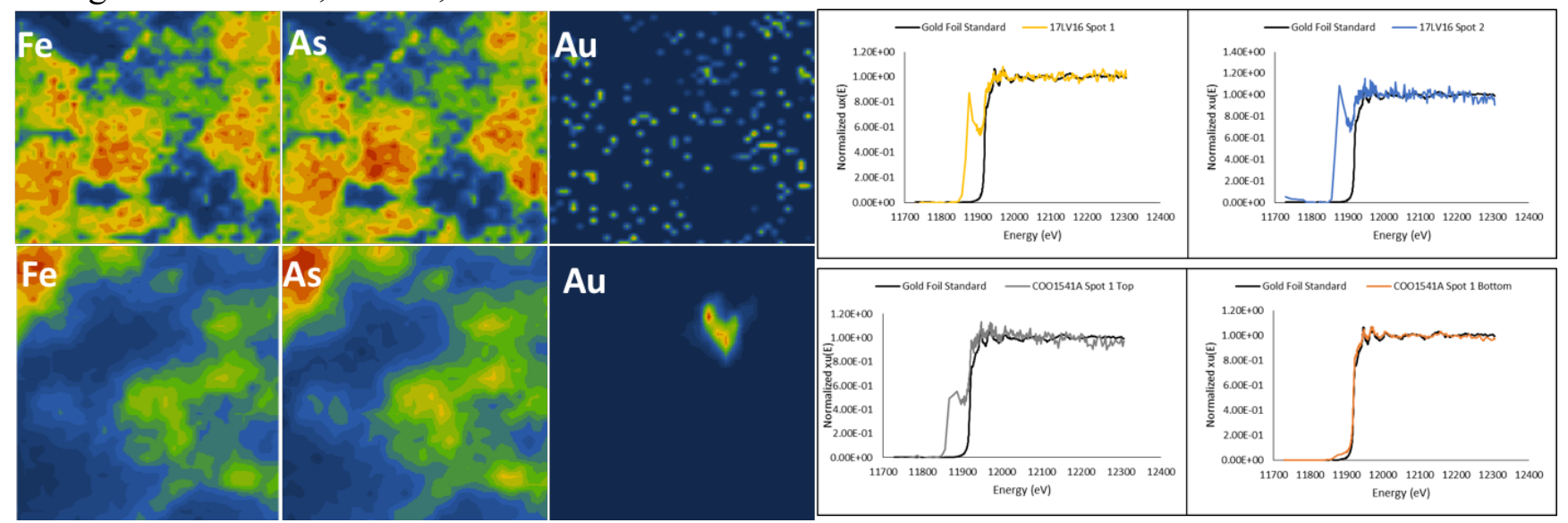

Figure 1. SR- $\mu$ XRF maps of Fe, As, and $\mathrm{Au}$ (left) and $\mathrm{Au}_{3}$-edge XANES spectra (right) for sample 17-LV-16 (top row) and C001541A (bottom row) collected at the VESPERS beamline at the CLS. SRXRF maps are 500 by $500 \mu \mathrm{m}$ (top) and 295 by $335 \mu \mathrm{m}$ (bottom). All of the XANES spectra are consistent with metallic gold. The feature between $11870-11880 \mathrm{eV}$ is attributed to As. 